\title{
The effect of number of irrelevant stimulus dimensions, verbalization, and sex on learning biconditional classification rules
}

\author{
ARCHIBALD C. BOWER AND WILLIAM L. KING \\ DALHOUSIE UNIVERSITY
}

Ss received three successive problems employing different relevant attributes but based on the same abstract classification rule. Instructions indicated which attributes were relevant for each problem leaving only the type of classification rule to be leamed. Increasing the number of irrelevant dimensions from two to four increased difficulty for the first of the three problems. Requiring $S$ s to verbalize a reason for each choice before making it facilitated solution especially for Problem 1.

In a bidimensional classification learning problem, the class membership of each stimulus is contingent on the presence or absence of two stimulus attributes (e.g., redness, squareness) rather than just one. Haygood \& Bourne (1965) have recently analyzed conceptual problems of this type into two components: attribute identification, in which Ss in possession of the abstract classification rule, e.g., a disjunction, must seek the specific stimulus attributes relevant to solution; and rule learning, in which Ss previously informed as to which attributes are relevant, must discover the classification rule specifying the manner in which the relevant attributes determine the class membership of each stimulus. Studies employing the latter, rule learning, paradigm (Haygood \& Bourne, 1965; King, 1966) have shown that the relative difficulty of different bidimensional rules (e.g., conjunction, disjunction, or biconditional) is the same for attribute identification and rule learning instructions. Such a result suggests that the process of forming a conceptual rule is closely related to those processes entailed in identifying the relevant attributes with advance knowledge of the rule. Moreover, Haygood \& Bourne (1965) have also shown that Ss with knowledge of neither the rule nor the relevant attributes, beforehand, experience the same relative difficulty for the different rules as those with knowledge of either component.

In order to conclude that the relative difficulty of different rules, in rule learning tasks, is accurately evaluated, one must assume that Ss attend only to the relevant attributes specified by the instructions. Otherwise, he may be assumed to be searching for the attributes in addition to the rule, a situation which may be described as a form of complete learning. The effect of the rule difficulty component in complete learning tasks may well be masked by the difficulty of the attribute learning component and any interaction between them. The present study was conducted in order to test the assumption that Ss in a rule learning task do not attend to irrelevant attributes. Should this assumption be unwarranted, then existing data from rule learning situations will be shown to provide unreliable estimates of rule difficulty per se.

Whereas the difficulty of attribute identification and, by implication, complete learning problems has been shown to increase with the addition of irrelevant stimulus dimensions (Kepros \& Bourne, 1966), performance in rule learning problems should be unaffected by such a manipulation. If the instructions specify the relevant stimulus attributes, Ss should not attend to irrelevant stimulus attributes, remaining unaffected by their variation.

Half the Ss were required to verbalize hypotheses before making classification responses while the other half were not. By examining Ss' hypotheses, it was possible to ascertain whether they contained mention of irrelevant attributes. At the same time, it was possible to examine the effect of verbalization itself on performance. The rule to be learned was a biconditional in which the joint presence or joint absence of both relevant attributes denotes the positive class, and the presence of only one but not the other of the two attributes denotes the negative class.

Method

Eighteen men attending Dalhousie University and 18 attending nursing school served as Ss.

The stimuli were three groups of $35 \mathrm{~mm}$ slides, one composed of four, one of five, and one of six binary dimensions.

Each S classified a series of slides into two groups. He was first shown six stimuli and asked to describe them in order to ensure that $S$ and $E$ agreed with respect to the description of the stimuli. Next, he was shown the two response buttons labeled + and -, and informed that pressing the correct button would illuminate a green light and pressing the wrong button would illuminate a red one. The rate of presentation for the stimuli was determined by $S$, who activated the slide changer by remote control. The rule learning instructions indicated which two attributes were relevant to solution. These attributes were represented on $4 \times 6$ in. cards and remained In view during the duration of the problem. The instructions stressed that the absence of the relevant stimuli was as important as their presence.

The experimental design was a 2 by 2 by 3 repeated measures factorial. The variables were verbalization, sex, and number of irrelevant stimulus dimensions. The criterion was 16 successive correct responses. However, if $\mathrm{S}$ did not begin the criterion run within 160 


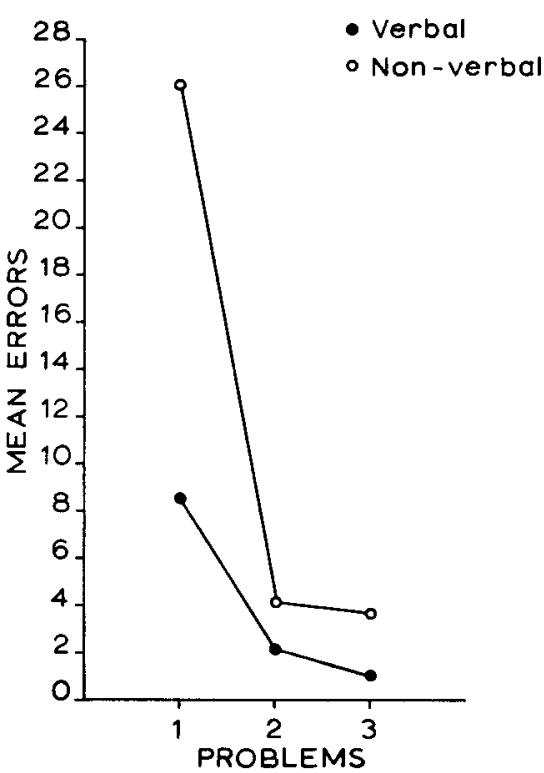

Fig. 1. Verbalization by problems.

trials, the solution was explained to him, and he was permitted an additional 24 trials during which to attain criterion. After each problem, $\mathrm{S}$ was asked to describe the principle which enabled him to classify the stimuli. Results

The Ss required to verbalize their hypotheses before obtaining feedback made significantly fewer errors than the others $(F=6.51, d f=1 / 24, p<.025)$. This result is graphically depicted in Fig. 1. The effects for problems and problems by dimensions were also significant ( $F=41.62$, df $=2 / 48, p<.005$ for problems and $F=3.64$, $\mathrm{df}=4 / 48, \mathrm{p}<.025$ for problems by dimensions). These results, depicted in Fig. 2, indicate that increasing the number of irrelevant stimulus dimensions increased the difficulty of Problem 1 but not of the remaining problems. Transfer was very nearly perfect from Problem 1 to the remaining two problems. Moreover, Ss mentioning at least one irrelevant attribute on Problem 1 made significantly more errors than those who did not according to a median test (Fisher's exact probability $=.005$ ). Three additional significant interactions with problems were obtained; however, all of them simply reflect the rate of transfer from Problem 1 and none of them qualify the previously-reported results.

The results suggest that Ss attend to irrelevant attributes at least for the first rule learning problem encountered, and that estimates of rule learning may be considerably influenced by attribute identification processes. Consequently, a comparison of rule difficulty under rule learning instructions will likely be confounded with difficulty due to attribute identification. The relative difficulty of different rules must be evaluated whlle

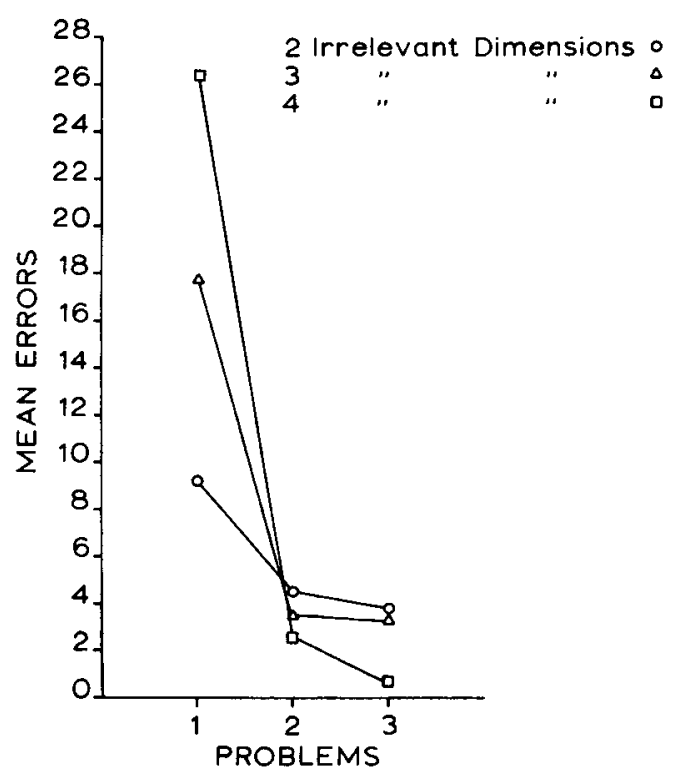

Fig. 2. Number of irrelevant dimensions by problems.

controlling for attention to irrelevant attributes; such an attempt is now in progress.

In all cases, Ss' explanations of the manner in which they solved the problems referred to the four attribute combinations of the relevant attributes, i.e., both present, the first present but not the second, the second present but not the first, and neither attribute present. Such evidence corroborates the analysis offered by Haygood \& Bourne (1965) that representations of these four combinations serve as mediators in rule learning problems. It is assumed that $S$ solves these problems by reducing the stimulus population to four subject-produced stimuli which may be easily associated with the two response classes. More supporting evidence for this interpretation comes from an analysis of the presolution error rates for each attribute combination treated as a separate problem. The average presolution error rate for each combination was .49 , virtually the same rate as predicted by chance. Thus, rule learning is characterized by the insightful solution of a series of subproblems and by extensive nonspecific transfer to subsequent problems of the same class

\section{References}

Haygood, R. C., \& Boume, L. E., Jr. Attribute and rule learning aspects of conceptual behavior. Psychol. Rev., 1965, 72, 175-195.

Kepros, P. C., \& Boume, L. E., Jr. Identification of biconditional concepts: effects of number of relevant and irrelevant dimensions. Canad. J. Psychol., 1966, 20, 198-207.

King, W. L. The learning and utilization of disjunctive and conjunctive classification rules: a developmental study. J. exp. child Psychol., 1966, 4, 217-231.

Note

1. This research was supported by Grant No. APA-124 from the National Research Council of Canada. 